\title{
In-vitro and in-vivo characterizations of novel modified starch transdermal drug delivery system
}

\begin{abstract}
Transdermal drug delivery systems are topically administered medicaments in the form of patches that deliver drugs for systemic effects at a predetermined and controlled rate. It works very simply in which drug is applied inside the patch and it is worn on skin for long period of time. Polymer matrix, drug, permeation enhancers are the main components of transdermal drug delivery systems. The objective of the present study was to develop the modified starch and 1,4-cis poly- butadiene nanoparticles as novel polymer matrix system. We have been studied the properties of a novel transdermal drug delivery system with clonidine as drug model.
\end{abstract}

Keywords: modified starch, 1,4-cis poly-butadienel, transdermal system
Volume 5 Issue 4 - 2017

\author{
Mohammadreza Saboktakin \\ Nanostructured Materials Synthesis Lab, NanoBMat Company, \\ Germany
}
Correspondence: Mohammadreza Saboktakin,
Nanostructured Materials Synthesis Lab, NANOBMAT
Company, GmbH, Hamburg, Germany,
Email Saboktakin123@gmail.com

Received: February 07, 2017 | Published: April 28, 2017

\section{Introduction}

Transdermal-patch technology has advanced tremendously since the first copolamine patch was introduced into the market in 1979. It can be attributed to today's advanced patch-making technology, through which nearly a billion patches are manufactured every year. ${ }^{1}$ These transdermal patches are classified into three types: drug in adhesive (the drug is directly dispersed into the adhesive polymer), reservoir (consists of a drug reservoir between a backing membrane and rate-controlling membrane, with a skin-contacting adhesive layer) and matrix (consists of a drug reservoir in the centre with a peripheral adhesive ring around the edges). ${ }^{2}$ Isosorbide dinitrate (ISDN) is commonly used for the therapy of stable angina pectoris and is traditionally administrated via oral or sublingual routes. However, loss of consciousness appears in patients when angina pectoris reaks out, and thus it is difficult for patients to take the medicine by themselves. Additionally, administrated orally ISDN has low bioavailability, owing to its high first-pass metabolism in the gastrointestinal tract and liver. Moreover, the critical point of antianginal therapy depends, to a certain extent, on the ability of the drug to produce an immediate effect. ${ }^{3-5}$ Thus, transdermal delivery may be an appropriate administration route for ISDN. Among the ISDN transdermal patches designed and reported previously, the drug-inadhesive patches are the simplest and the most commonly used design. Zhao et al. [6] developed single-layer drug-in- adhesive transdermal patches, in which the adhesive layer not only serves as an adhesion layer to the skin, but also is responsible for the release of the drug. The in vitro release results show that the release kinetics of ISDN is a first-order process, suggesting that the outwards moving of ISDN from the adhesive is associated with a passive diffusion process. Drug release from a drug-in-adhesive patch depends directly upon the drug concentration in the patch and follows first-order kinetics.

However, reservoir-type transdermal drug delivery could be observed the zeroorder kinetics. The rate controlling membrane, as a most important component in the reservoir-type transdermal patch, was responsible for controlling drug delivery. The rate-controlling membranes reported in previous publications included ethyl cellulose, ${ }^{6}$ collagen and chitosan, ${ }^{7}$ ethylene-vinyl acetate (EVA),${ }^{8}$ and acrylate polymers. ${ }^{9}$ Previous work in our lab has proven that acrylate polymers, as a new type of rate-controlling membranes, could control clonidine $\mathrm{HCl}$ solution release with zero order. ${ }^{10-15}$ But, such film-like acrylate polymers have not been applied in the production of patches to date.

\section{Materials and methods}

\section{Materials}

The cornstarch was purchased from Merck Company in Germany. 1,4-PBD was prepared from our previous research project and chlonidine drug with molecular weight 230Da or Catapres-TTs( Trade name) was purchased from Sigma Chemical company were dried using molecular sieve and then distilled in the presence of metallic sodium. Cobalt dialkylthiocarbamate as a main catalyst was purchased from Sigma-Aldrich.

\section{Instruments}

Melting points were obtained on a Mel-Temp melting point apparatus. Analytical TLCs were run on commercial Merck plates coated with silica gel GF250 ( $0.25 \mathrm{~mm}$ thick). The amount of released drug was determined on a Philips PU 8620 UV spectrophotometer at the absorption maximum of the free drug in aqueous alkali, using a $1 \mathrm{~cm}$ quartz cell. The nanoparticle samples were obtained by Freeze dryer. Model FD-10 (Pishtaz Engineering Company). The samples were examined to determine the mean diameter and size distribution. The powder morphology nanoparticles in the form of pellets (to measure grain size) was investigated using Philips XL-30 E SEM scanning electron micro-scope (SEM) at $30 \mathrm{kv}$ (max.). The samples were prepared by physical vapor disposition method. The gold layer thickness was about $100^{\circ} \mathrm{A}$ at these samples. Mooney viscometer, Shimadzu SMV-201, was used for Mooney viscosity characterization and measurements.

\section{Optical microscopy}

Optical microscopic technique was applied for the analysis of microstructure of the blend and composite samples. The magnified images of the samples were obtained using a microscope digital camera Leica DM 2500 M. Optical image of composites was carried out at room temperature. The maximum magnification obtained with the optical microscope was about 100. 


\section{Preparation of carboxymethylstarch (CMS)}

Firstly, the $0.5 \mathrm{~g}$ corn starch and $120 \mathrm{~mL} 2$-propanol were placed in a $500 \mathrm{ml}$ vessel and stirred for $2 \mathrm{~h}$. The $5 \mathrm{~g}$ sodium hydroxide was added and reacted for $1 \mathrm{~h}$ at $78-80^{\circ} \mathrm{C}$. After that, the $10 \mathrm{~g}$ chloroacetic acid was added to the vessel and stirred for another 2 hat $50^{\circ} \mathrm{C}$. The product was filtered and washed several times with ethanol, then dried under vacuum. The resulting CMS was crushed in a mortar [degree of substitution $(\mathrm{DS})=0.49]$.

\section{Preparation of CMS-I,4-PBD nanoparticles with clonidine}

Nanoparticles $(50 \mathrm{mg})$ and clonidine $(10 \mathrm{mg})$ were dispersed with stirring in $25 \mathrm{~mL}$ deionized water. After approximately $180 \mathrm{~min}$, the sample was sprayed into a liquid nitrogen bath cooled down to $77 \mathrm{~K}$, resulting in frozen droplets. These frozen droplets were then put into the chamber of the freeze-dryer. In the freeze drying process, the products are dried by a sublimation of the water component in an iced solution.

\section{Measurement of the rate of Clonidine release from nanoparticles}

A definite weight range of 10-15mg of PMPs was cut and placed in a $1.5 \mathrm{~mL}$ capacity micro centrifuge tube containing $1 \mathrm{ml}$ of release medium of the following composition at $37^{\circ} \mathrm{C}$ : phosphate buffered saline (140mM, pH 7.4) with $0.1 \%$ sodium azide and $0.1 \%$ Tween 80 . At predetermined time points, $100 \mu \mathrm{L}$ of release medium was sampled with replacement to which $3 \mathrm{~mL}$ of scintillation cocktail was added and vortexed before liquid-scintillation counting. The cumulative amount of clonidine released as a function of time was calculated.

\section{Drug content and encapsulation efficiency}

Encapsulation efficiency (EE) is the amount of added drug (\%) that is encapsulated in the formulation of the PMPs. The EE of drug from PMPs can be calculated in terms of the ratio of drug in the final formulation to the amount of added drug. An accurately weighed amount $(100 \mathrm{mg}$ ) of the formulation of PMPs was dispersed in $100 \mathrm{~mL}$ of Tris- $\mathrm{HCl}$ buffer. The sample was ultrasonicated for three consecutive 125 periods of $5 \mathrm{~min}$ each, with a resting period of $5 \mathrm{~min}$ each. It was left to centrifuge at $3000 \mathrm{rpm}$ for $15 \mathrm{~min}$. The concentration of clonidine in the decanted Tris- $\mathrm{HCl}$ buffer and two washing solutions were determined by measuring theabsorbance at $235 \mathrm{~nm}$ using a GBS Cintra 10-UV-vis spectrophotometer (Shimadzu, Japan).
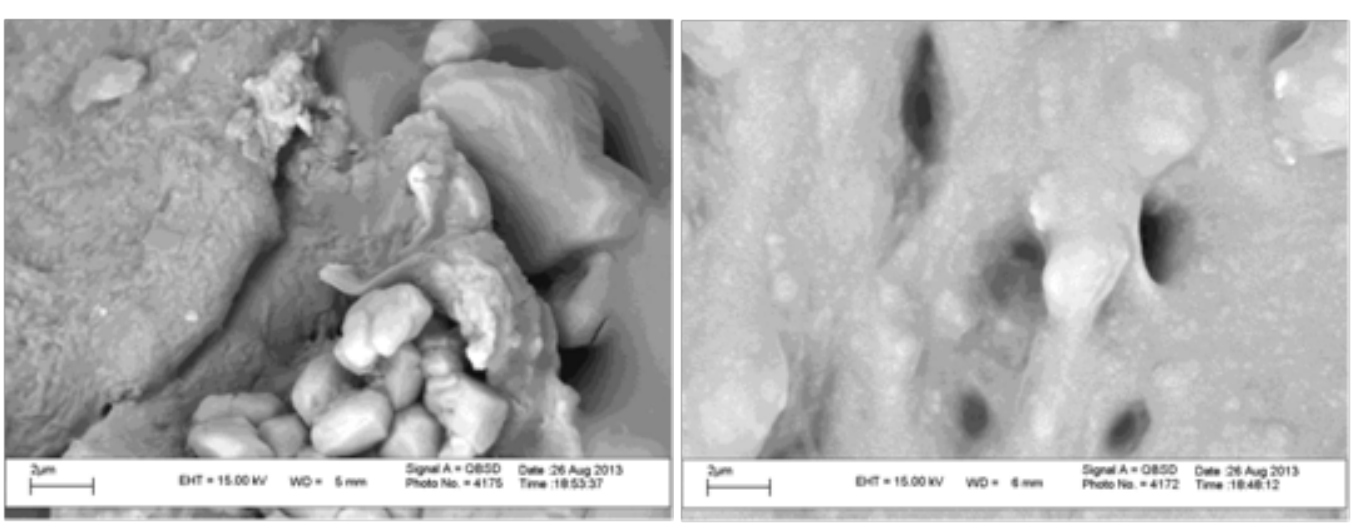

Figure I Morphology of clonidine loaded CMS-I,4-PBD polymer-matrix I 80 nanoparticle by emission scanning electron microscopy field.

\section{Particle size}

After drying at $37^{\circ} \mathrm{C}$ for $48 \mathrm{~h}$, the mean diameter of the dried PMPs was determined by a sieving method using USP standard sieves. Observations are recorded.

\section{The measurement of intrinsic viscosity}

The intrinsic viscosity $[\eta]$ was calculated through extrapolating $\ln \eta_{\mathrm{sp}} / \mathrm{C}$ when $\mathrm{C} \rightarrow 0$ where $\eta_{\mathrm{sp}}$ is the specific viscosity and $\mathrm{C}$ is the solution concentration in $\mathrm{g} / \mathrm{dL}$. Viscosity of diluted solutions (SV) of CMS-PBD nanoparticles were determined according to the ASTMD445 standard method using Ubbelohde viscometer and a solution concentration of $0.2 \mathrm{~g} / \mathrm{dL}$ in toluene solvent at $25^{\circ} \mathrm{C}$. Based on measurements, the intrinsic viscosity of typical CMS-PBD nanoparticles were found to be $[\eta]_{\text {lin }}=7.1 \mathrm{dL} / \mathrm{g}$. Mooney viscosity (MV) was measured according to ASTM D1646 standard method. The degree of branching of hyper branched PBD in polymer matrix was determined in two methods: by calculating the ratio of SV/MV and calculating on the basis of the numbers of linear, branched, and terminal units of macromolecules. Branching index of monomer units $\left(\mathrm{g}_{\mathrm{M}}\right)$ was determined by calculating the ratio of intrinsic viscosity of a branched polymer to that of a linear polymer, $g_{M}=[\eta]_{b r} /[\eta]_{\operatorname{lin}}$. Branching index of for linear PBD is $\mathrm{g}_{\mathrm{M}}=1.0$ and for branched PBD is about $\mathrm{g}_{\mathrm{M}}<1.0$.

\section{Results and discussion}

\section{Test results of electron microscope (SEM)}

The clonidine loaded CMS-1,4-PBD PMPs were characterized by FESEM for their size and distribution. Data showed that PMPs have a solid and near consistent structure. These PMPs have good spherical geometry. Figure 1 shows a representative SEM image of clonidine PMPs. Also, the results show that the surface of CMS-1,4-PBD PMPs shrank and a densely cross-linked gel structure was formed. The retardation of drug release from matrices of higher crosslinker content. The average drug entrapment was found to be $92.18 \pm 0.10 \%$ in the PMPs. The size of CMS-1,4-PBD PMPs was estimated by scanning electron microscopy (SEM) of the dried PMPs dispersions. The determination of PMPs size by SEM under a dry state does not result in an accurate absolute value of the hydrated PMPs size in dispersion, but only visualizes size range and particle shape. The incorporation of clonidine into the CMS-1,4-PBD PMPs produced a smooth surface and compact structure. The CMS-1,4-PBD PMPs are hydrophilic and would be expected to swell in water, thus producing a large hydrodynamic size when measured by the Zetasizer. The particle size of PMPs is measured. 


\section{Drug content and encapsulation efficiency}

Loading content of clonidine-PMPs with different theoretical loading densities were analyzed. Drug loading density, loading efficiency, and clonidine and PMPs yields of the PMPs with 5\%,10\%, $20 \%$ theoretical loading densities were calculated. Results in Figure 2 show that clonidine was effectively loaded into CMS-1,4-PBD PMPs, with high loading efficiency in all formulations. Clonidine loading densities for $5 \%, 10 \%$ and $20 \%$ theoretical loading were $4.6 \pm 0.1 \%$, $9.8 \pm 0.2 \%, 16.8 \pm 0.3 \%$, respectively. Net loading efficiencies of $5,10 \%$ theoretical loading were quantitative, whereas that of $20 \%$ theoretical loading was $89.26 \pm 1.5 \%$. The clonidine and PMPs yields were also sufficiently high to confirm PMPs encapsulation of clonidine as an effective strategy for solubilization of PS.

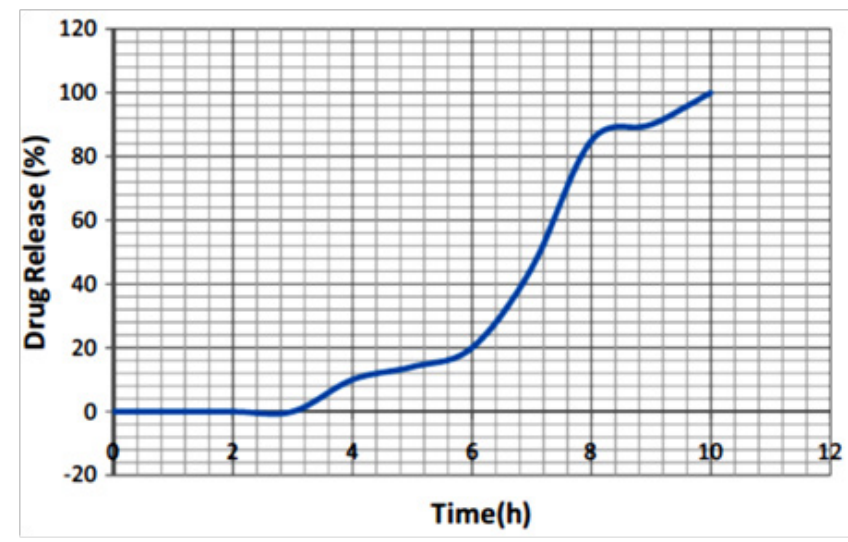

Figure 2 Release of Clonidine loaded CMS-I,4-PBD polymer - matrix 201 nanoparticle at 37.

\section{GPC measurement}

Based on the results, the weight average molecular weight of $\mathrm{M}_{\mathrm{w}}=3.0641 \times 105$, number average molecular weight of $\mathrm{Mn}=1.1175 \times 105$ and molecular mass distribution (poly dispersity) of $\mathrm{D}=\mathrm{M}_{\mathrm{w}} / \mathrm{M}_{\mathrm{n}}=2.7419$ were obtained using GPC method. The quantity of clonidine was determined using a standard calibration curve obtained under the same conditions. The primary mechanism for release of clonidine from matrix systems in vitro is swelling, diffusion, and disintegration. In addition, diffusion of clonidine may have been hindered by increased tortuosity of polymer accompanied by a swelling mechanism (Figure 3).

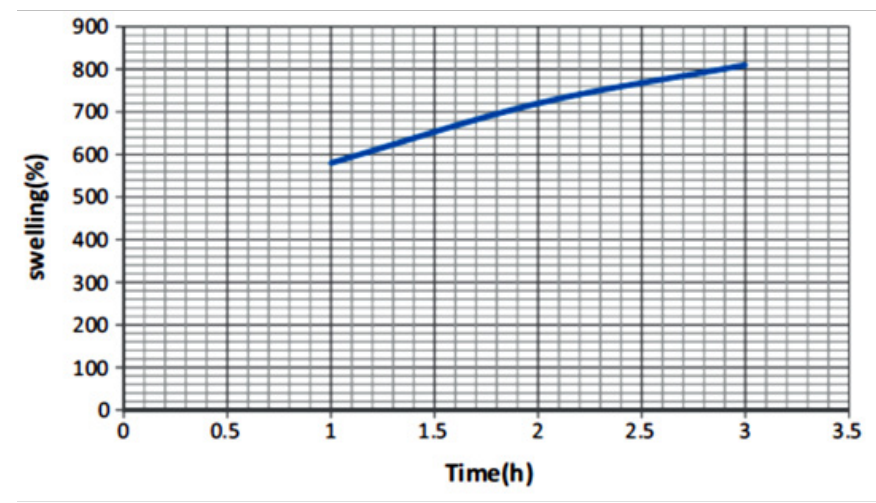

Figure 3 Time-dependent swelling behavior of Clonidine loaded CMS- I,4cis-219 PBD nanoparticles at $37^{\circ} \mathrm{C}$.

\section{In vitro release studies}

Nano polymer bonded clonidine $(50 \mathrm{mg}$ ) was poured into $3 \mathrm{~mL}$ of aqueous buffer solution ( $\mathrm{pH}$ 7.4). The mixture was introduced into a cellophane membrane dialysis bag. The bag was closed and transferred to a flask containing $20 \mathrm{~mL}$ of the same solution maintained at $37^{\circ} \mathrm{C}$. The external solution was continuously stirred, and $3 \mathrm{~mL}$ samples were removed at selected intervals. The triplicate samples were analyzed by UV spectrophotometer, and the quantity of clonidine was determined using a standard calibration curve obtained under the same conditions.

\section{In-vivo biological activity studies}

As shown in Figure 4, a single dose of $30 \mu \mathrm{g}$ hydrogel release $15 \mathrm{~min}$ after injection and reduced serum compared with control group in rats.

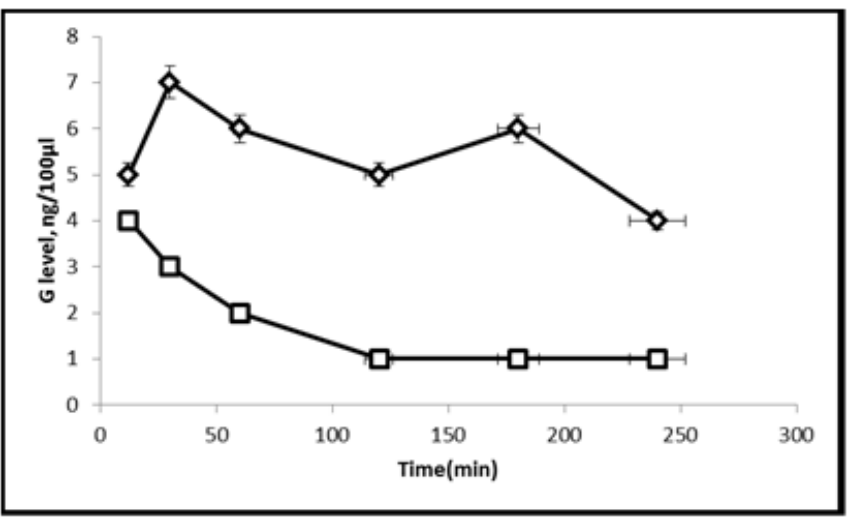

Figure $4 \mathrm{G}$ level of hydrogel release for time.

In-vivo release profile of drug from the in situ gel forming system

Serum concentration-time profiles of system after subcutaneous injection of the in situ gel forming system and solution are shown in Figure 5.

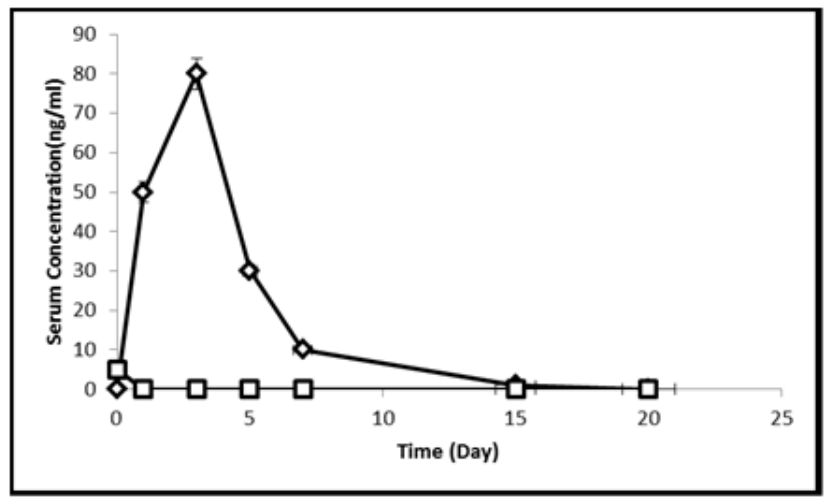

Figure 5 Serum concentration vs. time.

\section{Histological Analysis}

The clinical setting, the introduction of a drug delivery system under the renal capsule should preferably be none or minimally invasive. The synthesized hydrogel was implanted under the capsule of rat kidneys by injection, a process that required the generation of a sub capsular pocket by gently detaching the capsule from the renal cortex (Figure 6). ${ }^{16,17}$ 


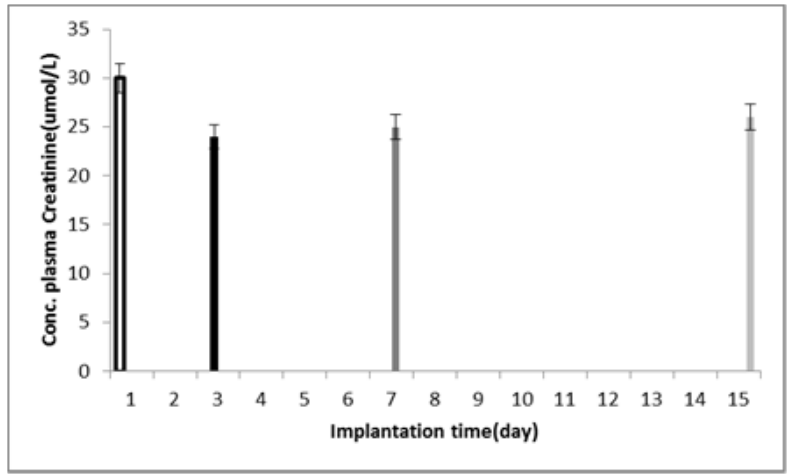

Figure 6 Concentration plasma creatinine versus implantation time.

\section{Conclusion}

Transdermal drug delivery is hardly an old technology, and the technology no longer is just adhesive patches. Due to the recent advances in technology and the incorporation of the drug to the site of action without rupturing the skin membrane accepted route of drug administration. The objective of the present study was to develop the modified starch and 1,4-cis poly-butadiene nanoparticles as novel polymer matrix system. We have been studied the in vitro and in-vivo properties of a novel transdermal drug delivery system with clonidine drug as model.

\section{Acknowledgements}

None.

\section{Conflict of interest}

The author declares no conflict of interest.

\section{References}

1. Chen GL, Shi XZ, Zhang ZH, et al. Study on percutaneous therapeutic system of isosorbide dinitrate effect of penetration enhancers on in vitro permeability through rat skin. Chin J Pharm. 1992;23(10):449-451.

2. Fung HL. Nitrate formulations and drug delivery systemsan overview. $Z$ Kardiol. 1985;4:4-9.

3. Gabiga H, Cal K, Janicki S. Effect of penetration enhancers on isosorbide dinitrate penetration through rat skin from a transdermal therapeutic system. Int J Pharm. 2000;199(1):1-6.

4. Johnson KI, Gladigau V, Schnelle K. Relationship between the pharmacodynamics and pharmacokinetics of two oral sustained-release formulations of isosorbide dinitrate in normal man. Arzneim Forsch. 1981;31(6):1026-1029.
5. Lewis S, Pandey S, Udupa. Design and evaluation of matrix type and membrane controlled transdermal delivery systems of nicotine suitable for use in smoking cessation. Indian J Pharm Sci. 2006;68(2):179-184.

6. Ng SF, Rouse JJ, Sanderson FD, et al. Validation of a static Franz diffusion cell system for in vitro permeation studies. Aaps Pharmscitech. 2010;11(3):1432-1441.

7. Ochalek M, Podhaisky H, Ruettinger HH, et al. SC lipid model membranes designed for studying impact of ceramide species on drug diffusion and permeation, Part III:Influence of penetration enhancer on diffusion and permeation of model drugs. Eur J Pharm Biopharm. 2012;82(2):360-366

8. Prausnitz MR, Langer R. Transdermal drug delivery. Nat Biotechnol. 2008;26(11):1261-1268.

9. Schmook FP, Meingassner JG, Billich A. Comparison of human skin or epidermis models with human and animal skin in in-vitro percutaneous absorption. Int J Pharm. 2001;215(1-2):51-56.

10. Teng $\mathrm{S}, \mathrm{Xu} \mathrm{H}$, Weng $\mathrm{W}$, et al. Development of a reservoir-type 342 transdermal delivery system containing eucalyptus oil for tetramethylpyrazine. Drug Deliv. 2013;20(1):19-24.

11. Subedi RK, Oh SY, Chun MK, et al. Recent advances in transdermal drug delivery. Arch Pharm Res. 2010;33(3):339-351.

12. Tan HS, Pfister WR. Pressure-sensitive adhesives for transdermal drug delivery systems. Pharm Sci Technol. 1999;2(2):60-69.

13. Taylor T, Chasseaud LF, Major RM, et al. Bioequivalence of a sustainedrelease isosorbide 350 dinitrate formulation at steady-state. Biopharm. Drug Dispos. 1985;6(2):119-129.

14. Thacharodi D, Rao KP. Rate-controlling biopolymer membranes as transdermal delivery systems for nifedipine:development and in vitro evaluations.biomaterials. 1996;17(13):1307-1311.

15. United States Pharmacopeial Convention. USP. USP 32 NF 27: United States Pharmacopeia [and] National Formulary. USA: United States Pharmacopeial Convention; 2009. p. 2712-2718.

16. Quintanilla YV, Verliefde A, Kim T, et al. Artificial neural network modes based on QSAR for predicting rejection of neutral organic compounds by polyamide nanofiltration 361 and reverse osmosis membranes. $J$ Membrane Sci. 2009;342(1-2):251-262.

17. Zhan X, Chen S, Tang JK, et al. poly (2- 363 hydroxy-3phenoxypropylacrylate, 4-hydroxybutyl acrylate, diethyl maleate) 364 membrane controlled clonidine linear release in the transdermal drug delivery 365 system. Eur Polym J. 2007;43(4):1588-1594. 